\title{
Utilisation du mélange de la pepsine avec la préparation microbienne "Fromase " dans la fabrication des fromages Edam et Kortowski
}

\author{
par \\ I. JARMUL*, A. REPS*, S. POZNANSKI* \\ et H. ZELAZOWSKA*
}

\section{INTRODUCTION}

La réduction de l'abattage des veaux a causé un déficit considérable en présure, ce qui a conduit à des recherches ayant pour l'objet de remplacer la présure par d'autres préparations enzymatiques.

On a constaté que la pepsine provenant des estomacs de porc, mélangée à la présure $(50 / 50)$ peut être utilisée avec succès pour la fabrication du fromage.

Cependant, le manque de présure devenant de plus en plus sensible, il est de plus en plus difficile d'en assurer la quantité suffisante pour en faire le mélange avec la pepsine.

De nombreux pays se voient contraints d'importer la présure ou ses succédanés, d'où leur intérêt pour des nouvelles possibilités d'utiliser la pepsine pour la fabrication du fromage.

Une de ces possibilités peut être l'utilisation de la pepsine en mélange avec des succédanés microbiens de présure. Les essais de GREen et Stockpoole [5] ont démontré que l'utilisation du mélange de pepsine avec la préparation "Noury », produite à partir de la moisissure Mucor pusillus v. Lindt, pour la fabrication du fromage Cheddar était plus efficace que l'utilisation de ces préparations séparément.

\footnotetext{
* Institut du Génie et de la Biotechnologie Alimentaire de l'Université Agrotechnique d'Olsztyn (Pologne).
} 
HUSEK et TEPLY [6] suggèrent la possibilité d'utiliser le mélange de la pepsine avec la préparation "Microzyme ", produite par Bacillus subtilis, pour la production des fromages à pâte molle.

Un des meilleurs succédanés de présure est la préparation « Fromase », produite en France par la Société « Rapidase ».

L'objectif de ces essais a été de déterminer la composition optimale du mélange de la pepsine de porc avec la préparation «Fromase " pour la production des fromages Edam et Kortowski (fromage polonais à pâte demi-molle).

\section{PROTOCOLE EXPERIMENTAL}

Dans des laiteries spécialisées dans la fabrication des fromages Edam et Kortowski, des fromages étaient fabriqués à l'échelle industrielle, en faisant coaguler le lait par la présure (activité de coagulation de $1: 10000$ ), la pepsine (activité protéolytique de 1:4000) et par le mélange de pepsine et de "Fromase » (activité de coagulation 1 : 100000 ), dans les proportions suivantes : $85 / 15,70 / 30$ et $50 / 50$, calculées en activité de coagulation. Des fromages expérimentaux et témoins (fabriqués avec la présure et la pepsine seules) étaient affinés dans des conditions convenables pour le fromage en question.

Après 4,8 et 12 semaines d'affinage pour le fromage Edam et après 2,4 et 6 semaines pour le fromage Kortowski, l'examen organoleptique était fait, et le degré de dégradation des protéines était établi, en déterminant la teneur en azote précipité avec des ions calcium [11], en azote soluble à pH 4,6 [15], en azote non-protéique [13], en azote peptidique [1] et en azote acides aminés [16]. En outre, les protéines de fromage étaient analysées par chromatographie sur le gel Sephadex G-100 [14, 17] et par électrophorèse sur le gel de polyacrylamide $[2,8]$.

\section{RESULTATS ET DISCUSSION}

Pour utiliser la pepsine, seule ou en mélange avec la "Fromase", pour faire coaguler le lait, aucun changement des paramètres techniques n'a été nécessaire dans la fabrication des fromages Edam et Kortowski.

Aucune différence n'a été constatée dans la cinétique de la coagulation par la présure, la pepsine et le mélange "pepsine-Fromase ».

La fermeté du caillé-pepsine était un peu moins élevée, dans le cas des types de fromage fabriqués, que celle du caillé présure, ou 
celle du caillé produit par le mélange « pepsine-Fromase ». Ces observations sont en accord avec les résultats précédents de PoZNANSKI et al. [10] et de RePs et al. [12].

En analysant le rendement des matières azotées du lait, on a constaté le taux moins élevé de ces matières, passées dans le lactosérum, en comparaison avec la fabrication avec la présure, quand la pepsine était employée pour la fabrication des fromages analysés (tab. 1). On sait qu'au $\mathrm{pH}$ du lait de fromagerie, l'activité de la pepsine est moins élevée que celle de la présure.

En utilisant le mélange " pepsine-Fromase » pour fabriquer le fromage Edam, le degré d'utilisation des matières azotées était identique à celui observé au cours de la fabrication du fromage avec la pepsine seule (tab. 1).

\section{TABLEAU 1}

Taux des matières azotées passés du lait au lactosérum au cours de la fabrication du fromage

\begin{tabular}{|c|c|c|c|c|c|}
\hline \multirow{2}{*}{$\begin{array}{r}\text { Sorte de préparation } \\
\text { coagulante }\end{array}$} & \multirow{2}{*}{ Présure } & \multirow{2}{*}{ Pepsine } & \multicolumn{3}{|c|}{ Mélange des préparations } \\
\hline & & & P-F $85 / 15$ & P-F $70 / 30$ & P-F $50 / 50$ \\
\hline $\begin{array}{l}\text { Sorte } \\
\text { de fromage }\end{array}$ & \multicolumn{5}{|c|}{$\mathrm{N}$ du lactosérum / $\mathrm{N}$ du lait en pourcentage } \\
\hline Edam & 25,72 & 24,27 & 24,27 & 24,33 & 24,66 \\
\hline Kortowski & 26,95 & 25,65 & 25,85 & 26,09 & 27,80 \\
\hline \multicolumn{2}{|l|}{$\mathrm{P}=$ Pepsine. } & \multicolumn{3}{|c|}{$\mathrm{F}=$ "Fromase $»}$. & \\
\hline
\end{tabular}

Au cours de la fabrication du fromage Kortowski, le taux des matières azotées, passées du lait dans le lactosérum, augmentait légèrement, quand augmentait la quantité de "Fromase " mélangée à la pepsine (tab. 1).

On sait que la nature et l'intensité des modifications des protéines est un des indices pour déterminer le degré d'affinage du fromage. En suivant l'évolution de la protéolyse dans le fromage par la teneur en matières azotées précipitées par les ions calcium, en 
matières azotées solubles à $\mathrm{pH} 4,6$, non protéiques, peptidiques et en azote acides aminés (tab. 2), on a observé que la dégradation des protéines s'effectuait plus lentement dans les fromages Edam et Kortowski fabriqués avec de la pepsine, que dans les fromages fabriqués avec la présure. La différence de la teneur en formes diverses des matières azotées, entre le fromage à la présure et celui à la pepsine, était plus considérable pour le fromage Edam que pour le fromage Kortowski. Cela peut s'expliquer par des températures plus élevées qu'on applique au cours de la fabrication du fromage Edam, ce qui n'est pas sans effet, comme on le sait, sur l'inactivation de la pepsine $[4,10]$.

Dans le fromage à la pepsine, des protéines de masse moléculaire élevée, de 141000 à 80000 , étaient observées en plus grande quantité que dans le fromage à la présure (tab. 3). Par exemple, après la durée d'affinage, prévue par la norme de qualité, il y avait 50 p. 100 de protéines de masse moléculaire élevée dans le fromage Edam, fabriqué à l'aide de la pepsine, et 30 p. 100 seulement dans le fromage à la présure. De même, dans le fromage Kortowski âgé de 4 semaines, et fabriqué avec la pepsine, le taux des protéines de masse moléculaire élevée était de 70 p. 100 environ, tandis qu'il était de 50 p. 100 pour la fabrication à la présure.

Dans le fromage Edam (tab. 2) fabriqué avec le mélange " pepsine-Fromase $"$, le degré de dégradation des protéines était semblable à celui du fromage à la présure (excepté le fromage fabriqué avec le mélange de $85 / 15$, dont l'affinage s'effectuait pareillement à celui du fromage à la pepsine). Par contre, dans le fromage Kortowski, une protéolyse plus intense était observée pour le mélange que pour la présure (tab. 2).

En analysant la répartition des protéines de fromage sur le gel Sephadex G-100, on a constaté, pareillement aux études des autres auteurs [7, 17] que les différences dans la nature de la dégradation des protéines, qu'on observe entre les fromages de diverses sortes, ainsi que les différences observées dans la même espèce, dépendaient de la préparation coagulante utilisée.

On a constaté (tab. 3) que le degré des protéines du fromage Edam et Kortowski, fabriqué avec du mélange " pepsine-Fromase » s'approchait de celui du fromage à la pepsine.

Dans les types de fromage analysés, le même nombre de fractions protéiques était observé, ayant des masses moléculaires approchantes. Le pourcentage de ces fractions était pareil. Cependant, dans le fromage Kortowski âgé de 6 semaines, fabriqué avec la pepsine, quatre fractions de protéines ont été trouvées, tandis que le fromage avec le mélange "pepsine-Fromase " en avait deux seulement. De même, dans le fromage à la pepsine, le pourcentage des protéines à masse moléculaire élevée dans le fromage avec le mélange " pepsine-Fromase " était supérieur à celui du fromage à la présure. 
TABLEAU 2

Degré de dégradation des protéines au cours de l'affinage du fromage

\begin{tabular}{|c|c|c|c|c|c|c|c|c|c|c|c|c|c|c|c|c|c|}
\hline \multirow{2}{*}{$\begin{array}{l}\text { Sorte de } \\
\text { fromage }\end{array}$} & \multirow{2}{*}{\multicolumn{2}{|c|}{$\begin{array}{l}\text { Sorte de préparation } \\
\text { coagulante }\end{array}$}} & \multicolumn{3}{|c|}{$\begin{array}{l}\mathrm{N} \text { précipité } \\
\text { avec } \mathrm{Ca}^{++}\end{array}$} & \multicolumn{3}{|c|}{$\mathrm{N}$ soluble à $\mathrm{pH} 4,6$} & \multicolumn{3}{|c|}{$\mathrm{N}$ non protéique } & \multicolumn{3}{|c|}{$\mathrm{N}$ de peptides } & \multicolumn{3}{|c|}{$\mathrm{N}$ d'acides aminés } \\
\hline & & & \multicolumn{15}{|c|}{ en pourcentage de $\mathrm{N}$ total } \\
\hline \multicolumn{3}{|c|}{ Durée d'affinage en semaines } & 4 & 8 & 12 & 4 & 8 & 12 & 4 & 8 & 12 & 4 & 8 & 12 & 4 & 8 & 12 \\
\hline \multirow{5}{*}{ Edam } & & résure & 86,98 & 83,24 & 76,40 & 12,80 & 16,70 & 23,59 & 5,64 & 8,47 & 13,53 & 1,44 & 2,15 & 3,63 & 2,90 & 5,74 & 6,55 \\
\hline & & epsine & 88,70 & 86,15 & 81,00 & 10,28 & 13,78 & 18,99 & 4,58 & 7,88 & 8,41 & 1,12 & 1,25 & 1,20 & 1,48 & 3,57 & 3,98 \\
\hline & \&ै ฮี & P-F $85 / 15$ & 87,90 & 85,38 & 80,40 & 10,37 & 14,09 & 19,54 & 5,07 & 8,55 & 8,67 & 1,24 & 1,53 & 1,75 & 2,50 & 4,24 & 5,32 \\
\hline & 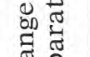 & P-F 70/30 & 86,50 & 83,40 & 78,60 & 10,95 & 16,33 & 21,26 & 5,53 & 8,68 & 10,10 & 1,34 & 1,76 & 2,14 & 2,64 & 5,02 & 5,56 \\
\hline & $\sum^{\mathbb{0}}$ & P-F $50 / 50$ & 85,86 & 82,38 & 77,90 & 11,67 & 17,44 & 21,90 & 5,78 & 9,72 & 11,17 & 1,33 & 1,84 & 2,18 & 2,73 & 5,24 & 5,65 \\
\hline \multicolumn{3}{|c|}{ Durée d'affinage en semaines } & 2 & 4 & 6 & 2 & 4 & 6 & 2 & 4 & 6 & 2 & 4 & 6 & 2 & 4 & 6 \\
\hline \multirow{5}{*}{ Kortowski } & \multirow{2}{*}{\multicolumn{2}{|c|}{$\begin{array}{l}\text { Présure } \\
\text { Pepsine }\end{array}$}} & 86,34 & 83,52 & 81,11 & 15,20 & 16,13 & 17,80 & 6,34 & 8,38 & 9,89 & 1,61 & 2,05 & 2,56 & 6,11 & 6,33 & 7,29 \\
\hline & & & 87,45 & 84,13 & 82,61 & 13,24 & 15,94 & 17,14 & 4,19 & 5,89 & 8,16 & 0,86 & 1,44 & 2,20 & 5,08 & 6,31 & 7,24 \\
\hline & \multirow{3}{*}{ 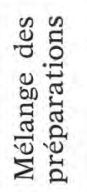 } & P-F 85/15 & 85,64 & 82,82 & 80,34 & 15,07 & 17,67 & 19,48 & 6,65 & 8,60 & 9,11 & 1,56 & 2,10 & 2,66 & 6,20 & 6,94 & 7,47 \\
\hline & & P-F 70/30 & 84,69 & 82,03 & 79,89 & 16,01 & 18,14 & 20,03 & 7,02 & 9,41 & 10,84 & 1,63 & 2,14 & 2,72 & 6,80 & 7,00 & 7,50 \\
\hline & & P-F $50 / 50$ & 82,73 & 81,89 & 79,12 & 17,30 & 18,70 & 20,89 & 7,45 & 9,61 & 11,63 & 1,79 & 2,50 & 3,21 & 7,01 & 7,73 & 8,05 \\
\hline \multicolumn{3}{|l|}{$P=$ Pepsine } & \multicolumn{6}{|c|}{$F=«$ Fromase ». } & & & & & & & & & \\
\hline
\end{tabular}


TABLEAU 3

Caractéristique de la répartition chromatographique des protéines du fromage sur le gel Sephadex G-100

\begin{tabular}{|c|c|c|c|c|c|c|c|c|c|c|c|c|}
\hline & & \multicolumn{11}{|c|}{ Sortes de fromages } \\
\hline \multirow{4}{*}{\multicolumn{2}{|c|}{$\begin{array}{l}\begin{array}{c}\text { Sorte } \\
\text { de }\end{array} \\
\text { préparation } \\
\text { coagulante }\end{array}$}} & \multicolumn{5}{|c|}{ Edam } & \multicolumn{6}{|c|}{ Kortowski } \\
\hline & & \multirow{3}{*}{ 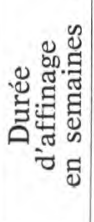 } & \multicolumn{4}{|c|}{$\begin{array}{l}\text { Masse moléculaire des protéines contenues } \\
\text { dans le pic } \times 10^{-3}\end{array}$} & \multirow{3}{*}{ 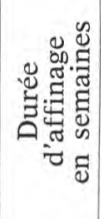 } & \multicolumn{5}{|c|}{$\begin{array}{c}\text { Masse moléculaire des protéines contenues } \\
\text { dans le pic } \times 10^{-3}\end{array}$} \\
\hline & & & $126 \leqslant M \leqslant 147$ & $74 \leqslant M \leqslant 111$ & $37 \leqslant M \leqslant 74$ & $17 \leqslant M \leqslant 25$ & & $86 \leqslant M \leqslant 108$ & $32 \leqslant M \leqslant 40$ & $23 \leqslant M \leqslant 29$ & $17 \leqslant M \leqslant 20$ & $10 \leqslant M \leqslant 14$ \\
\hline & & & & $\mathrm{N}$ du pic/N & élue (p. 100) & & & & $\mathrm{N} d u$ & pic / $\mathrm{N}$ élue & (p. 100) & \\
\hline \multicolumn{2}{|c|}{ Présure } & $\begin{array}{r}4 \\
8 \\
12\end{array}$ & $\begin{array}{r}8,38 \\
32,02 \\
17,06\end{array}$ & $\frac{23,85}{\overline{18,94}}$ & $\begin{array}{l}41,95 \\
50,65 \\
49,72\end{array}$ & $\begin{array}{l}26,34 \\
17,32 \\
14,28\end{array}$ & $\begin{array}{l}2 \\
4 \\
6\end{array}$ & $\begin{array}{l}57,46 \\
48,39 \\
49,67\end{array}$ & $\overline{-}$ & $\begin{array}{r}17,54 \\
15,86 \\
8,99\end{array}$ & $\begin{array}{l}\overline{14,51} \\
12,21\end{array}$ & $\begin{array}{l}25,00 \\
21,24 \\
19,49\end{array}$ \\
\hline \multicolumn{2}{|c|}{ Pepsine } & $\begin{array}{r}4 \\
8 \\
12\end{array}$ & $\begin{array}{l}47,17 \\
43,84 \\
48,24\end{array}$ & $\begin{array}{r}35,38 \\
6,52 \\
30,15\end{array}$ & $\begin{array}{l}-\overline{30,43} \\
12,13\end{array}$ & $\begin{array}{r}17,43 \\
19,20 \\
9,45\end{array}$ & $\begin{array}{l}2 \\
4 \\
6\end{array}$ & $\begin{array}{l}80,10 \\
72,86 \\
74,58\end{array}$ & $\frac{-}{-}$ & $\begin{array}{l}7,40 \\
6,63 \\
6,63\end{array}$ & $\begin{array}{r}- \\
10,66 \\
9,37\end{array}$ & $\begin{array}{r}12,50 \\
9,85 \\
9,69\end{array}$ \\
\hline \multirow{3}{*}{ 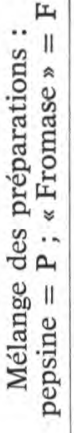 } & P-F 85/15 & $\begin{array}{r}4 \\
8 \\
12\end{array}$ & $\begin{array}{l}47,30 \\
40,00 \\
50,19\end{array}$ & $\begin{array}{l}30,37 \\
16,13 \\
26,74\end{array}$ & $\begin{array}{l}- \\
27,95 \\
12,27\end{array}$ & $\begin{array}{l}22,31 \\
15,90 \\
10,78\end{array}$ & $\begin{array}{l}2 \\
4 \\
6\end{array}$ & $\begin{array}{l}76,84 \\
73,87 \\
87,50\end{array}$ & $\begin{array}{c}\overline{9,32} \\
-\end{array}$ & $\begin{array}{c}9,24 \\
4,83 \\
-\end{array}$ & $\frac{-}{-}$ & $\begin{array}{l}13,92 \\
11,98 \\
12,50\end{array}$ \\
\hline & P-F 70/30 & $\begin{array}{r}4 \\
8 \\
12\end{array}$ & $\begin{array}{l}34,20 \\
34,03 \\
34,38\end{array}$ & $\begin{array}{l}36,30 \\
15,48 \\
14,23\end{array}$ & $\begin{array}{c}- \\
27,73 \\
26,49\end{array}$ & $\begin{array}{l}23,48 \\
22,75 \\
24,90\end{array}$ & $\begin{array}{l}2 \\
4 \\
6\end{array}$ & $\begin{array}{l}77,56 \\
74,62 \\
88,50\end{array}$ & $\begin{array}{c}6,95 \\
7,75 \\
--\end{array}$ & $\begin{array}{c}\overline{6,93} \\
-\end{array}$ & $\frac{-}{-}$ & $\begin{array}{l}15,49 \\
10,70 \\
11,50\end{array}$ \\
\hline & P-F 50/50 & $\begin{array}{r}4 \\
8 \\
12\end{array}$ & $\begin{array}{l}37,17 \\
26,32 \\
46,58\end{array}$ & $\begin{array}{l}46,15 \\
18,80 \\
16,81\end{array}$ & $\begin{array}{c}- \\
41,15 \\
24,29\end{array}$ & $\begin{array}{l}16,68 \\
13,71 \\
12,28\end{array}$ & $\begin{array}{l}2 \\
4 \\
6\end{array}$ & $\begin{array}{l}77,59 \\
74,61 \\
86,64\end{array}$ & $\begin{array}{c}4,50 \\
- \\
-\end{array}$ & $\begin{array}{c}\overline{9,04} \\
-\end{array}$ & $\begin{array}{c}\overline{7,63} \\
-\end{array}$ & $\begin{array}{r}17,91 \\
8,72 \\
13,26\end{array}$ \\
\hline
\end{tabular}


La quantité de la préparation «Fromase " dans le mélange ne paraissait exercer aucune influence significative sur le caractère de la dégradation des protéines. Pour le fromage Edam, fabriqué avec le mélange de $70 / 30$, la teneur en protéines de masse moléculaire la plus basse s'est montrée supérieure à celle du fromage avec le mélange de $85 / 15$ ou $50 / 50$.

Pour le fromage Kortowski âgé de 2 semaines (tab. 3), la teneur en protéines de masse moléculaire moyenne diminuait à mesure que le taux de la préparation "Fromase " augmentait tandis que les protéines de poids moléculaire le plus bas augmentaient en même temps. Pourtant, après 4 et 6 semaines d'affinage du fromage Kortowski, aucune différence significative n'était observée dans la teneur en protéines des diverses fractions indépendamment du taux de la préparation enzymatique.

Les répartitions électrophorétiques des protéines de fromages Edam et Kortowski sur le gel de polyacrylamide (fig. 1, 2) démontraient des différences dans la spécificité d'action des préparations coagulantes enzymatiques que nous avons étudiées.

Sur les électrophorégrammes de répartition des protéines des fromages Edam et Kortowski, fabriqués à l'aide de la présure et la pepsine, on a constaté, à la première étape de l'affinage, le même nombre de bandes de protéines et le même pourcentage. Cependant, on voyait le nombre des fractions de protéines augmenter à mesure que l'affinage progressait. Néanmoins, la densité des bandes indiquait que ces préparations enzymatiques dégradaient les protéines d'une façon un peu différente.

En analysant la répartition des protéines sur gel de polyacrylamide des fromages analysés, fabriqués à l'aide du mélange " pepsine-Fromase », on a constaté au début de l'affinage que le nombre des fractions augmentait avec le taux croissant de la préparation microbienne dans le mélange avec la pepsine. Pourtant, après 12 semaines d'affinage du fromage Edam, le même nombre de fractions de même densité était observé, indépendamment de la quantité de la préparation «Fromase ». De même, les électrophorégrammes du fromage Kortowski, âgé de 6 semaines et fabriqués avec des mélanges à $85 / 15$ et $70 / 30$ montraient un nombre égal des fractions. Pourtant, la densité de ces fractions était différente.

En analysant des électrophorégrammes de la répartition des protéines sur gel de polyacrylamide pour le fromage Edam (fig. 1) après 4 semaines d'affinage, on a constaté des bandes plus nombreuses dans la région de la caséine $\alpha$ que dans la région de la caséine $\beta$, ce qui peut indiquer que la pepsine et son mélange avec la «Fromase " dégradaient la fraction caséine $\alpha$ plus fortement, comme le fait la présure $[3,9]$.

De même, de l'analyse des densités des bandes, qui se présentaient sur l'électrophorégramme du fromage Kortowski (fig. 2), fabri- 


\section{पIIIIII] UIIIII \\ [IIIIIIIII III

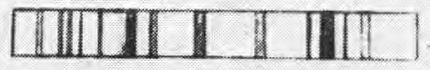

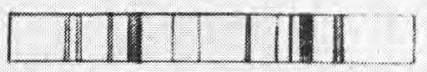
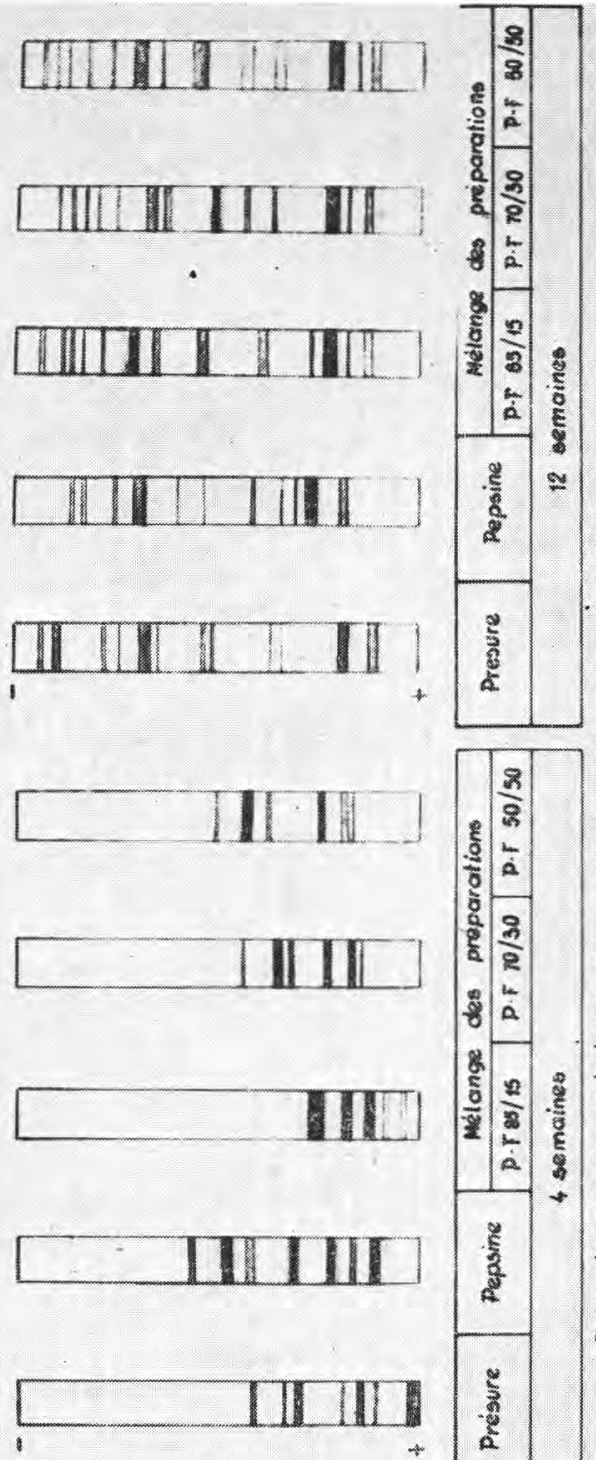

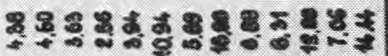

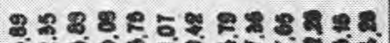

คั๙

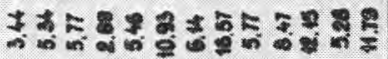

ง ำ

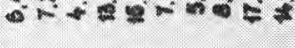

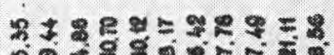

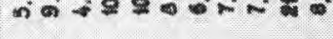

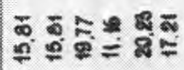

กำ ำ

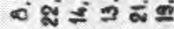

$728 \div 8$

สำ

ธ $\delta$ \%

है

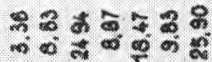
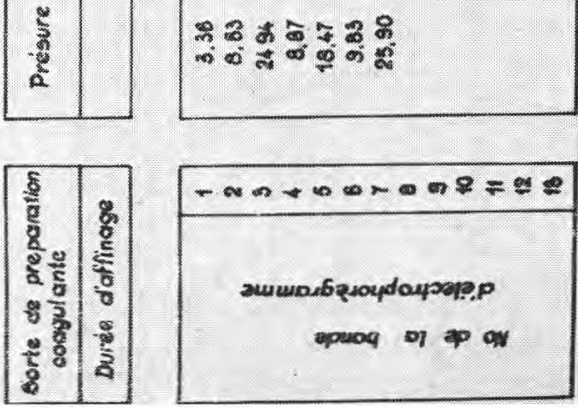

fig. 1

Répartition électrophorétique des protéines du fromage Edam sur le gel de polyacrylamide $(\mathrm{P}=$ Pepsine ; $\mathrm{F}=$ Fromase $)$. 


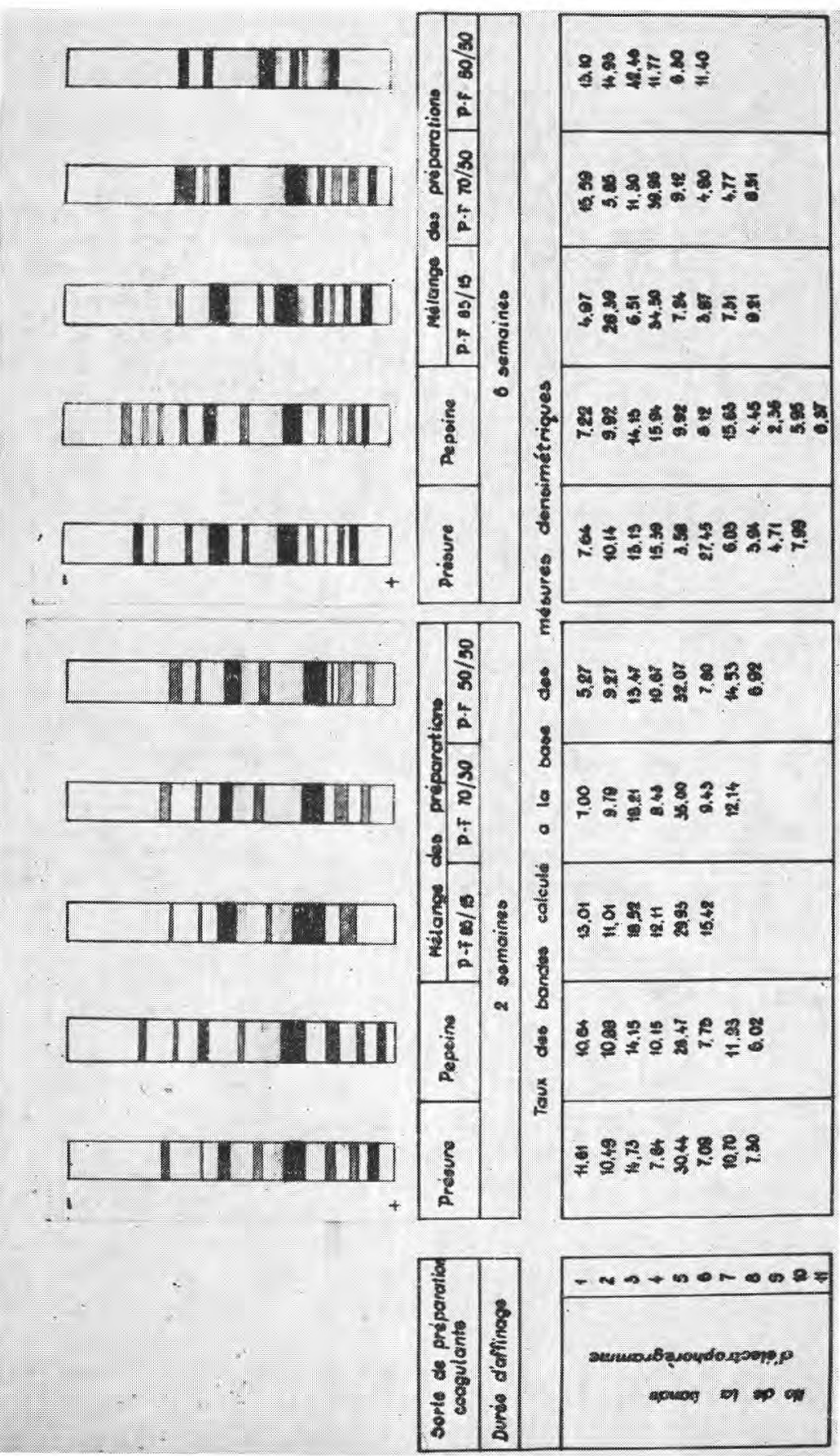

fig. 2

Répartition électrophorétique des protéines du fromage Kortowski sur le gel de polyacrylamide $(\mathrm{P}=$ Pepsine $; \mathrm{F}=$ Fromase $)$. 
qué avec la présure et la pepsine, il apparaît que l'étendue de la bande correspondant à la caséine $\alpha$ diminuait au cours de l'affinage. Après 2 semaines d'affinage du fromage Kortowski avec le mélange " pepsine-Fromase ", on a observé que les bandes dans la région de la caséine $\alpha$ devenaient plus nombreuses à mesure que le taux de la préparation microbienne augmentait dans le mélange.

Néanmoins, dans le fromage fabriqué avec le mélange " pepsineFromase ", la fraction caséine $\beta$ était plus dégradée qu'elle ne l'était dans le fromage à la présure, ce qui est prouvé par des bandes plus nombreuses dans la région de la fraction caséine $\beta$, comme on le voit sur l'électrophorégramme du fromage Edam, âgé de 12 semaines, fabriqué à l'aide du mélange et aussi par la coloration moins intense de la bande, qui correspondait à la caséine $\beta$ dans le cas du fromage Kortowski.

Les fromages Edam et Kortowski, fabriqués à l'aide du mélange " pepsine-Fromase » ont été examinés organoleptiquement par un pannel d'experts qui ont confirmé la possibilité d'obtenir un produit ayant des propriétés typiques pour le fromage en question, dans le temps prévu par la norme de qualité. On a constaté qu'en utilisant le mélange de la pepsine avec la "Fromase » on peut fabriquer un fromage dont la qualité est même meilleure que celle du fromage à la présure et surtout que celle du fromage fabriqué avec la pepsine seule. Après la durée d'affinage prévue par la norme de qualité, le fromage fabriqué avec le mélange se caractérisait par une saveur et une odeur supérieures.

L'examen organoleptique des types de fromage analysés, ainsi que les résultats des analyses chimiques et physico-chimiques ont démontré, que l'usage du mélange " pepsine-Fromase " à $50 / 50$ ou $70 / 30$ était le plus profitable pour la fabrication des fromages à pâte dure.

Pour des fromages à pâte molle, il suffit déjà de remplacer la pepsine par 15 p. 100 de "Fromase" pour obtenir un fromage de bonne qualité.

\section{Rés u mé}

On a étudié l'utilité de la pepsine de porc, en mélange avec la préparation "Fromase ", pour la fabrication des fromages Edam et Kortowski (fromage polonais à pâte demi-molle).

On a analysé les paramètres techniques de la fabrication du fromage, le rendement en matières azotées et le degré de dégradation des protéines au cours de l'affinage.

Pour faire coaguler le lait, le mélange "pepsine-Fromase " s'est montré le plus efficace dans les proportions 50/50 ou 70/30 (exprimées en activité de coagulation) pour la fabrication du fromage 
Edam, et dans les proportions $85 / 15$ pour la fabrication du fromage Kortowski.

\section{S u m m a r y}

\section{USING PEPSIN IN MIXTURE WITH MICROBIAL RENNET KORTOWSKI «FROMASE» IN CHEESE-MAKING OF EDAM AND KORTOWSKI}

Studies were made on the utility of pig pepsin, in mixture with "Fromase» preparation for the manufacture of Edam and Kortowski (Polish semi-hard cheese) cheese.

Technical parameters of cheese manufacture, utilization of cheese nitrogen compounds, and the process of protein degradation in the course of cheese ripening were analysed.

The most efficent "pepsin-Fromase» mixture to be used for milk protein coagulation was found to be that of $50 / 50$ or $70 / 30$ (exprimed as coagulation activity) for the Edam cheese manufacture and that of $85 / 15$ for the Kortowski cheese manufacture.

Reçu pour publication en avril 1981.

\section{Bibliographie}

[1] Boulanger (P.) et Biserte (G.) (1949). - Chromatographie de partage des amino-acides libres du plasma sanguin. Bull. Soc. Chimie biol., 31, 696.

[2] DAvis (B. J.) (1964). - Disc electrophoresis. II. Method and application to human serum proteins. Ann. New York Acad. Sc., 121, 404.

[3] Desmazeaud (M. J.) and Gripon (J. C.) (1977). - General mechanism of protein breakdown during cheese ripening. Milchwiss., 32, 731.

[4] GREen (M. L.) (1972). - Assessment of swine, bovine and chicken pepsins as rennet substitutes for Cheddar cheese-making. J. Dairy Res., 39, 261.

[5] Green (M. L.) and Stackpoole (A.) (1975). - The preparation and assessment of a suitable Mucor pusillus Lind proteinase - swine pepsin mixture for Cheddar cheese-making. J. Dairy Res., 42, 297.

[6] HuseK (V.), and TePly (M.) (1978). - Biochemical properties of microbial proteases in the mixture with rennets of animal origin. XX Int. Dairy Congr., 439.

[7] Jedrychowski (L.), Poznanski (S.), Jakubowski (J.), Smietana (Z.) und KrefFt (R.) (1975). - Einsatz von Enzympräparaten mikrobiellen Ursprungs zur kontinuierlichen Käseherstellung. Milchwiss., 30, 668.

[8] Ornstein (A. A.) (1964). - Disc electrophoresis. I. Background and theory. Ann. New York Acad. Sc, 121, 321.

[9] Phelan (J. A.), Guiney (J.) and Fox (P. F.) (1973). - Proteolysis of $\beta$-casein in Cheddar cheese. J. Dairy Res., 40, 105.

[10] Poznanski (S.), Reps (A.) et Smietana (Z.) (1969). - Otrzymywanie, charakterystyka i proby zastosowania ekstraktu pepsyny do koagulacji mleka przy wyrobie sera. Zesz. nauk. WSR Olszt., 25, 321. 
[11] Poznanski (S.) and Rymaszewski (J.) (1965). - Proteolysis during the ripening of Edam cheese with the participation of some bacteria strains. I. Changes in particular nitrogen fractions. Milchwiss., 20, 14.

[12] Reps (A.), Poznanski (S.), Babuchowski (A.) et Jedrychowski (L.) (1979). Propriétés des substituts de présure fabriqués à partir de Mucor miehei. Le Lait, 59, 1,

[13] Schober (R.), Niclaus (W.) und Christ (W.) (1961). - Anwendung der «Finger-Abdruck-Methode» auf die Kennzeichnung von Käsesorten durch ihre proteolytischen Inhaltsstoffe. Milchwiss., 16, 140.

[14] SEPHADEX. Gel filtration in theory and practice (1970). - Pharmacia Fine Chemicals.

[15] SoDE-Mogensen (T.) (1948). - Determination of the degree proteolytic decomposition in cheese with special reference to the formed titration. Meddelande, No 21, Fran Statens Majeriförsök, Alnarp - Akarp.

[16] Stadhouders (J.) (1960). - The hydrolysis of protein during the ripening of dutch cheese. The enzyme and bacteria involved. Neth. Milk Dairy J., $14,83$.

[17] Tокiтa (F.) and Hosono (A.) (1968). - An investigation about Limburger cheese ripening by gel filtration on Sephadex. Milchwiss., 23, 758. 